\title{
Quantum Gravity
}

\author{
Konstantinos Patrinos \\ National Technical University of Athens, Athens, Greece \\ Email:kpatr@central.ntua.gr
}

How to cite this paper: Patrinos, K. (2021) Quantum Gravity. Journal of Modern Physics, 12, 1447-1463.

https://doi.org/10.4236/jmp.2021.1210087

Received: July 29, 2021

Accepted: August 21, 2021

Published: August 24, 2021

Copyright $\odot 2021$ by author(s) and Scientific Research Publishing Inc. This work is licensed under the Creative Commons Attribution International License (CC BY 4.0).

http://creativecommons.org/licenses/by/4.0/

\begin{abstract}
Quantum gravitational theory, based on the hypothesis of the absolute reference system, reveals the function of the effects of the gravitational field at the microscopic and macroscopic scale. The quantum nature of gravitational potential, and the dynamics and kinetic energy of photons and elementary particles under the influence of the gravitational field are studied, and a quantum interpretation of gravitational redshift is given. There is also a complete agreement of this quantum gravitational theory with the existing experimental data.
\end{abstract}

\section{Keywords}

Quantum Gravity, Gravitational Time Dilation, Gravitational Redshift, Perihelion of Mercury, Gravitational Deflection of Light, Time Delay of Light

\section{Introduction}

Quantum gravitational theory based on hypothesis of the absolute reference system is fully compatible with quantum mechanics and quantum field theory, for the following reasons:

1) Quantum gravity based on the hypothesis of the absolute reference system does not need the possibility of renormalization, since there are no infinities arising in calculated quantities. There is also no point-particle, since the elementary particles of matter have a specific structure, consisting of bound photons.

2) Changes in the operating rate of clocks under the influence of a gravitational field or due to motion at high speeds comparable to the speed of light in vacuum, based on the hypothesis of an absolute reference system, are not related to the concept of spacetime, but are related to the influences exerted on the structural elements of the particles, such as the effect of a gravitational field on bound photons, as well as Lorentz contraction. 
3) Spacetime is not dynamic as in the general theory of relativity. The space of the hypothesis of the absolute reference system is a Euclidean space, and there is the possibility of an unambiguous description of all the cosmic events observed in the absolute reference system.

The study of inertial systems based on the hypothesis of the absolute reference system is stated in [1] [2] [3]. In the present work, the quantum nature of the gravitational field is studied and in addition the effects of the gravitational field on physical phenomena are studied by supplementing what is already stated in [4].

Some references related to the general theory of relativity, in which are presented the principles of this gravitational theory by its author himself, are in [5]-[10].

\section{Quantum Description of the Gravitational Field}

Based on the hypothesis of the absolute reference system, the elementary photonic wave is considered to be an oscillator whose mean value of kinetic energy is equal to $(1 / 2) h v$, where $h$ is Plank's constant and $v$ is the frequency, and mean value of dynamic energy is also equal to (1/2) $h v$ ([2], subsection 3.3. Harmonic Oscillator). The total energy of a photon is equal to $h v$. Also according to the section "Introduction to Particle Mechanics" in [1], we have the relation $h v=m_{p h} c^{2}$, where $m_{p h}$ is the photonic mass and $c$ is the speed of light in vacuum. We must then consider the effect of the gravitational field on a photon coming from a space in which there is no gravitational field, and then propagates in a gravitational field, that is, on the already disturbed ether.

We consider that outside the gravitational field is a body or a measuring instrument, when it is included in the natural reference frame of the celestial body to which the gravitational field is due, but it is very far from this celestial body, so that the effect of the gravitational field is considered practically zero. Also, we denote by $m_{p h_{o}}, c_{o}$ and $v_{o}$ the mass, velocity and frequency of a photon, when it is outside the gravitational field, while these physical quantities will be denoted by $m_{p h}$, c and $v$ respectively, when the same photon is inside the gravitational field.

The momentum of the photon, without the presence of a gravitational field, making use of the clock which is outside the gravitational field, is given by the relation $p_{p h_{o}}=m_{p h_{o}} c_{o}$ ([1] section 4. Introduction to Particle Mechanics). The corresponding relation for a photon propagating in a gravitational field and resulting from the use of the aforementioned clock, in vector form, is:

$$
\boldsymbol{p}_{p h}=m_{p h} \boldsymbol{c}
$$

where $m_{p h}, \boldsymbol{c}$ are functions dependent on the radius $r$ ([4] section 2. Mass and velocity of a photon in the gravitational field). We consider that the photon propagates in space with the simultaneous presence of a gravitational field derived from the spherical body of mass $M$.

Therefore, the momentum - energy relation of a photon that is a force carrier 
of the gravitational field is:

$$
\frac{\boldsymbol{p}_{p h}^{2}}{m_{p h}}=\mathcal{E}_{p h}
$$

Suppose that in a small volume $\delta V$, at a radial distance $r$, a set of $N$ force carriers of the gravitational field is an elementary part of the whole spherical wave of all force carriers of the gravitational field of a celestial spherical body of mass $M$. The wavefunction $\Psi$ of this system of $N$ photons of different frequencies will obey the classical differential equation of a spherical electromagnetic wave. Specifically, the action of the kinetic energy operator ${ }^{1}$ on the wavefunction $\Psi$ is given by the equation:

$$
\hat{\mathcal{E}}_{p h, k i n} \Psi=\frac{i \hbar}{2} \frac{\partial \Psi}{\partial t}
$$

and gives us the equation of action of the total energy operator on the wavefunction $\Psi$ of the photon:

$$
\hat{\mathcal{E}}_{p h} \Psi=2 \hat{\mathcal{E}}_{p h, k i n} \Psi=i \hbar \frac{\partial \Psi}{\partial t}
$$

The momentum operator is $\hat{\boldsymbol{p}}_{p h}=-i \hbar \nabla$. From Equations (1) and (2) we get the equation:

$$
\boldsymbol{p}_{p h}^{2}=\frac{1}{c^{2}} \mathcal{E}_{p h}^{2}
$$

from which we get the classical electromagnetic wave equation:

$$
\left(\nabla^{2}-\frac{1}{c^{2}} \frac{\partial^{2}}{\partial t^{2}}\right) \Psi(r, t)=0
$$

where the wavefunction $\Psi$ depends on the radial distance $r$ and not on the vector position $\boldsymbol{r}$, since this wave is spherical. Under these conditions the action of the operator $\nabla^{2}$ on the wavefunction $\Psi$ is expressed by the equation:

$$
\nabla^{2} \Psi=\frac{1}{r^{2}} \frac{\partial}{\partial r}\left(r^{2} \frac{\partial \Psi}{\partial r}\right)
$$

so, Equation (6) can take the form:

$$
\frac{\partial^{2}(r \Psi)}{\partial r^{2}}-\frac{1}{c^{2}} \frac{\partial^{2}(r \Psi)}{\partial t^{2}}=0
$$

The solution of this differential equation, for $N$ photons, which are force carriers in the elementary volume $\delta V$, is given by the relation:

$$
\Psi(r, t)=\frac{1}{r} \sum_{i=1}^{N} A_{i} \mathrm{e}^{i\left(k_{i} r-\omega_{i} t\right)}
$$

where $A_{i}$, for $i=1,2, \cdots, N$, is constant. The gravitational potential, $U(r)$, is proportional to the time-averaged absolute value of $\Psi(r, t)$, so it is in a form given by the relation:

${ }^{1}$ The momentum and kinetic energy operators are described in the literature reference [2], subsection 2.2, Wave Function of a Free Particle, Equations (2.9) and (2.10). 


$$
U(r) \sim \overline{|\Psi|}=\overline{\sqrt{\Psi \Psi^{*}}}=\frac{A}{r}
$$

where $A=\sqrt{\sum_{i=1}^{N}\left|A_{i}\right|^{2}}$. If we denote by $\boldsymbol{E}$ the intensity of the gravitational field (something analogous to the intensity of the electric field in electromagnetism), then this is given by the relation:

$$
\boldsymbol{E}(r)=-\nabla U(r)
$$

On a photon located in the above elementary volume $\delta V$ and having mass $m_{p h}$ a gravitational force is exerted, determined by the equation:

$$
\boldsymbol{F}=m_{p h} \boldsymbol{E}=-m_{p h} \nabla U=-\frac{G M m_{p h}}{r^{2}} \hat{u}_{r}
$$

where $\hat{u}_{r}$ is the unit radial vector. Therefore, the expression for gravitational potential, $U(r)$, is:

$$
U(r)=-\frac{G M}{r}
$$

We must also summarize the findings of the study regarding the dependence of the mass and velocity of a photon under the influence of a gravitational field, as stated in [4] (section 3, Mass and Velocity of a Photon in the Gravitational Field). This dependence of the velocity and mass of a photon on the radial distance $r$, which is the distance of the photon from the center of the spherical celestial body of mass $M$, is determined by the relations:

$$
\begin{gathered}
c=c_{o}\left(1-\frac{4 G M}{r c_{o}^{2}}\right)^{1 / 2} \\
m_{p h}=m_{p h_{o}}\left(1-\frac{4 G M}{r c_{o}^{2}}\right)^{-3 / 4}
\end{gathered}
$$

Since the elementary particles have as structural elements the bound photons, the function of the photonic mass, which is due to the dependency on the radial distance, will be analogous to the corresponding function of the mass of a body located inside the gravitational field. If $m$ and $m_{o}$ are the masses of a spherical body inside and outside the gravitational field respectively, and $r$ is the radial distance, the mass function $m$ is given by the relation:

$$
m=m_{o}\left(1-\frac{4 G M}{r c_{o}^{2}}\right)^{-3 / 4}
$$

The dynamic energy of a photon, due to the effect of the gravitational field when moving from a position outside the gravitational field (i.e. theoretically from infinite distance) to a radial distance $R$, is calculated by the integral:

$$
V_{p h}(R)=\int_{\infty}^{R} \boldsymbol{F} \cdot \mathrm{d} \boldsymbol{r}=-\int_{R}^{\infty}\left(-m_{p h} \nabla U(r)\right) \cdot \mathrm{d} \boldsymbol{r}
$$

With the help of the relation (11) and the relation (12) for the gravitational potential, we get: 


$$
\begin{aligned}
V_{p h}(R) & =\int_{R}^{\infty} \frac{G M}{r^{2}} m_{p h_{o}}\left(1-\frac{4 G M}{r c_{o}^{2}}\right)^{-3 / 4} \mathrm{~d} r \\
& =-m_{p h_{o}} c_{o}^{2}+m_{p h_{o}} c_{o}^{2}\left(1-\frac{4 G M}{R c_{o}^{2}}\right)^{1 / 4} \\
& =-m_{p h_{o}} c_{o}^{2}+m_{p h} c^{2}
\end{aligned}
$$

The first-order approximation of this photon gravitational dynamic energy is calculated according to relation:

$$
V_{p h}(R) \simeq-\frac{G M m_{p h_{o}}}{R}
$$

and respectively for a particle, which had mass $m_{o}$ when it was outside the gravitational field, and now it is inside the gravitational field at a radial distance $R$, the gravitational dynamic energy is calculated approximately according to relation:

$$
V(R) \simeq-\frac{G M m_{o}}{R}
$$

The nature of this dynamic energy is studied in [4], section 2, The Origin of Attractive Force in a Gravitational Field, and we observe that the energy $E_{p h}$, which is also stated in [4] (section 2), at a radial distance $r$ is equal to $-V_{p h}(r)$. Indeed, by putting $\boldsymbol{F}=f(r) \hat{u}_{r}$, as stated in [4] (section 2, Equation (2)), we get the relation:

$$
\boldsymbol{F}=f(r) \hat{u}_{r}=\frac{\mathrm{d} E_{p h}}{\mathrm{~d} r} \hat{u}_{r}=-\nabla V_{p h}(r)
$$

which is easily confirmed according to relation (17).

The total energy of a photon propagating inside a gravitational field at a radial distance $R$, using the measuring instruments of a laboratory located outside the gravitational field, according to relation (17) is:

$$
m_{p h} c^{2}=m_{p h_{o}} c_{o}^{2}+V_{p h}(R)
$$

where $m_{p h} c^{2}=h v, m_{p h_{o}} c_{o}^{2}=h v_{o}$, and the frequencies $v$ and $v_{o}$ are the frequencies of the same photon when it is inside the gravitational field or outside it respectively, measured by a clock located outside the gravitational field. As already mentioned at the beginning of this section, there is a term for the kinetic energy of this photon, which is $\frac{1}{2} h v_{o}=\frac{1}{2} m_{p h_{o}} c_{o}^{2}$, and two terms of dynamic energy, one of which is that of the elementary photonic harmonic oscillator equal to the kinetic energy term, and the other term of dynamic energy, $V_{p h}(R)$, is due to the effect of the gravitational field on the photon. Due to this effect of the gravitational field, a physical system located within a gravitational field becomes non-inertial. Therefore, in order to estimate the kinetic energy of the photon using the above-mentioned clock, one must calculate the quantity $\frac{1}{2} h v_{o}$, which is equal to the kinetic energy of the same photon, when this photon is outside the gravitational field. 
The spherical electromagnetic wave, which consists of free photons of gravitational field force carriers, comes from the continuous conversion of bound photons into free photons (and vice versa), and their frequency spectrum is the spectrum of the "mass frequency" of bound photons ${ }^{2}$ from which they originate. The density of these force carriers at a radial distance of $r$ is proportional to the time-averaged value of the quantity $\Psi \Psi^{*}$ (as mentioned in [2], subsection 2.2, Wave Function of a Free Particle), and is also proportional to the mass of the spherical homogeneous body from which the gravitational field originates. But the mass of the spherical homogeneous celestial body is affected by the gravitational field created by the body itself. Suppose that the whole mass of the spherical body is converted to free photons in a space in which there is no gravitational field and that this measured mass of free photons is equal to $M_{o}$. This mass, $M_{o}$, is called non-gravitational mass. Therefore the mass of the spherical body before this conversion, that is, when it was in a solid state, must have been slightly greater than the measured mass of free photons, due to the existence of the gravitational field created by the body itself. This mass, which we consider equal to $M$, is called gravitational mass.

If we want to calculate the gravitational mass, $M$, of this body we can do it with a good approximation ignoring terms of very small order of magnitude. Suppose first that a concentric spherical part of this body of radius $r$ and gravitational mass $\mathcal{M}(r)$ is surrounded by a spherical shell of differential thickness $\mathrm{d} r$ and differential gravitational mass $\mathrm{d} \mathcal{M}(r)$, as shown in Figure 1, and that the density $\rho$ of the non-gravitational mass is constant throughout the body. The differential gravitational mass of the spherical shell is given by the relation:

$$
\mathrm{d} \mathcal{M}(r)=\mathrm{d} \mathcal{M}_{o}(r)\left(1-\frac{4 G \mathcal{M}(r)}{r c_{o}^{2}}\right)^{-3 / 4}
$$

where $\mathrm{d} \mathcal{M}_{o}(r)$ is the non-gravitational differential mass of the spherical shell. By setting $\mathcal{M}(r)-\mathcal{M}_{o}(r)=\Delta \mathcal{M}$ we get the approximate relation:

$$
\frac{G \mathcal{M}(r)}{r c_{o}^{2}}=\frac{G \mathcal{M}_{o}(r)}{r c_{o}^{2}}+\frac{G \Delta \mathcal{M}}{r c_{o}^{2}} \simeq \frac{G \mathcal{M}_{o}(r)}{r c_{o}^{2}}
$$

since the quantity $G \mathcal{M}_{o}(r) /\left(r c_{o}^{2}\right)$ is quite small (of the order of $10^{-5}$ for the solar system). If we denote by $R$ the radius of the spherical body, the approximate calculation of the gravitational mass of the body gives us:

$$
M=\int_{0}^{R} \mathrm{~d} \mathcal{M}(r) \simeq \int_{0}^{R}\left(1+\frac{3 G \mathcal{M}_{o}(r)}{r c_{o}^{2}}\right) \mathrm{d} \mathcal{M}_{o}(r)
$$

Since the above density, $\rho$, is constant, it follows that the non-gravitational mass in the spherical part of radius $r$ is $\mathcal{M}_{o}(r)=\frac{4}{3} \pi \rho r^{3}$, and also $\mathrm{d} \mathcal{M}_{o}(r)=4 \pi \rho r^{2} \mathrm{~d} r$, so, it follows that using the previous integration, the gravitational mass of the body is determined by the following relation:

${ }^{2}$ The concept of mass frequency is described in [1], subsection 4.1, The Structure of the Smallest Elementary Particle. 


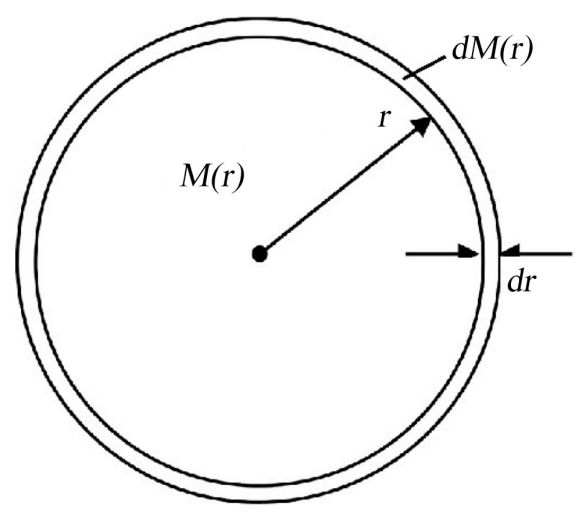

Figure 1. A concentric spherical part of the body of radius $r$ and gravitational mass $\mathcal{M}(r)$ is surrounded by a spherical shell of differential thickness $\mathrm{d} r$ and differential gravitational mass $\mathrm{d} \mathcal{M}(r)$.

$$
M=M_{o}\left(1+\frac{9}{5} \frac{G M_{o}}{R c_{o}^{2}}\right)
$$

where $M_{o}=\frac{4}{3} \pi \rho R^{3}$.

\section{Additional Effects of the Influence Exerted by the Gravitational Field on Matter}

As mentioned in the previous section, the kinetic energy of a photon propagating inside a gravitational field is equal to the kinetic energy of the same photon when it is outside the gravitational field. Also, the physical reference system of a massive celestial body $M$ is not an inertial reference system, since it dynamically affects all the structural elements of all the elementary particles of matter. According to the hypothesis of the absolute reference system, these structural elements are the bound photons.

Although all the internal dynamic energies of an elementary particle have a sum equal to zero in order for the particle to be in a stable state, the state of the particle due to the gravitational field remains as an exogenous non-inertial state of the particle. For example the effect on the velocity and mass of a captive photon, according to relations (13) and (14), gives us the total energy of the bound photon, but its kinetic energy is maintained equal to the kinetic energy estimated when this captive photon is outside the gravitational field. Therefore the kinetic energy of a particle is that which the particle had when it was outside the gravitational field, moving in an inertial system at a speed measured by the measuring instruments of the same inertial system equal to the velocity of the particle inside the gravitational field that measured by same measuring instruments. The kinetic energy of a particle which is at a radial distance $r$ and moves with speed $u$ measured by the measuring instruments of a laboratory located outside the gravitational field, according to the hypothesis of the absolute reference system, is 
given by the equation:

$$
E_{\text {kin }}=\frac{1}{2} m_{o} \gamma^{2} c_{o}^{2}-\frac{1}{2} m_{o} c_{o}^{2}=\frac{1}{2} m_{o} \gamma^{2} u^{2}
$$

where $\gamma=\left(1-u^{2} / c_{o}^{2}\right)^{-1 / 2}$.

A notable change, due to the effect of the gravitational field, is the change in the quantitative estimation of the electric charge of an elementary charged particle. As has already been proved by the principles of the hypothesis of the absolute reference system (in [1], subsection 2.4.2, The Charge and the Force Carrier of Electromagnetic Interactions, Equation (2.60)), a charged particle initially accelerated by electromagnetic interactions in the inertial reference system of the laboratory, then moving at a constant velocity $v$ with respect to it measured by the instruments of the laboratory, has an electric charge equal to $q_{v}$, while before acceleration, practically stationary in the laboratory, it had an electric charge equal to $q$. The corresponding relation is:

$$
q_{v}=\frac{q}{\gamma_{v}}
$$

A charged particle under the influence of a gravitational field, considered as stationary at a radial distance $r$, undergoes a change in the quantitative estimation of its charge due to the expression of the estimated time ${ }^{3}$, obtained by using a clock that is at the same radial distance, $r$, as that of the electric charge, according to relation:

$$
t_{g}=t\left(1-\frac{4 G M}{r c_{o}^{2}}\right)^{1 / 4}
$$

because this estimated time causes a change in the estimation of the emission rate of the force carriers in relation to the estimation of the emission rate of the force carriers resulting from a clock outside the gravitational field, as in the case of the aforementioned moving charged particle.

We denote by $q$ the electric charge of a particle located at a radial distance $R$ within the gravitational field, which is measured by the measuring instruments of a laboratory located outside the gravitational field. Also, we denote by $q_{o}$ the electric charge of the same particle when this particle is outside the gravitational field, measured by the aforementioned laboratory. The functional relation between the quantities $q$ and $q_{o}$ is:

$$
q=q_{o}\left(1-\frac{4 G M}{R c_{o}^{2}}\right)^{1 / 4}
$$

If the measuring instruments and the aforementioned charged particle are located inside the gravitational field, at a radial distance $R$, then the electric charge is evaluated as equal to $q_{o}$.

${ }^{3}$ The relation between the estimated time inside the gravitational field and the measured time with a clock outside the gravitational field, and the discussion regarding this relationship is in [4], section 4, Gravitational time dilation and redshift, Equation (21). 


\section{The Quantum Interpretation of Gravitational Redshift}

In order to study the phenomenon of gravitational redshift, we will study the energy emission spectrum derived from a hydrogen atom under the influence of a gravitational field, using the principles of quantum mechanics based on the absolute reference system hypothesis.

We consider a hydrogen atom affected by the gravitational field of a spherical and homogeneous celestial body of mass $M$, and located at a constant radial distance $R$ from the center of this spherical body. In order to study this phenomenon according to the principles of hypothesis of the absolute reference system, the energy of this atomic electron should be estimated using the clock of the particle under study, i.e. the clock of the electron of the hydrogen atom.

According to the relevant example in [2] (subsection 3.4, Hydrogen Atom, Equation (3.29)), if we use the subscript $o$ for frequencies, measured with the instruments of a laboratory which is outside the gravitational field, where the phenomenon we are considering takes place, the average value of kinetic energy at the energy level of quantum number $n$, is given by the equation:

$$
\overline{E_{k i n, n}}=\frac{1}{2} h n v_{n_{o}}
$$

In the same example (in [2], subsection 3.4, Hydrogen Atom, Equations (3.31), (3.33)), if we use the subscript $o$ for mass and electric charge measured with the instruments of the above laboratory, it appears that:

$$
\overline{E_{k i n, n}}=\frac{m_{o} e_{o}^{4}}{2 \hbar^{2}} \frac{1}{n^{2}}
$$

Therefore:

$$
\frac{1}{2} h n v_{n_{o}}=\frac{m_{o} e_{o}^{4}}{2 \hbar^{2}} \frac{1}{n^{2}}
$$

But we need to understand the origin of the first member of the Equation (27). The definition of particle frequency is given in [2] (subsection 2.1, Particle-Frequency and Wavelength, Equation (2.3)) by the relation $v_{q_{o}}=\sum_{i=1}^{N} v_{u_{i_{o}}}$, where $v_{u_{i}}$ is the frequency that comes from the kinetic energy of the $i$ bound photon. The kinetic energy of the particle is:

$$
E_{k i n}=\frac{1}{2} m_{o} \gamma^{2} u^{2}=\frac{1}{2} h \sum_{i=1}^{N} v_{u_{i_{o}}}
$$

Therefore the kinetic energy in relation to the particle frequency is:

$$
E_{k i n}=\frac{1}{2} m_{o} \gamma^{2} u^{2}=\frac{1}{2} h v_{q_{o}}
$$

Therefore the average value of kinetic energy, when $\gamma \simeq 1$, is given by the relation:

$$
\overline{E_{k i n}}=\frac{1}{2} m_{o} \overline{u^{2}}=\frac{1}{2} h \overline{v_{q_{o}}}
$$

where $\overline{v_{q_{o}}}=n v_{n_{o}}$, as formulated in [2] (subsection 2.4, Particle Motion in 
Closed Orbits, Equation (2.23)), and finally a general equation is:

$$
\overline{E_{k i n}}=\frac{1}{2} m_{o} \overline{u^{2}}=\frac{1}{2} h n v_{n_{o}}
$$

Suppose that the hydrogen atom moves slowly from a region outside the gravitational field to the celestial spherical body, that is, it gradually enters the gravitational field, and finally immobilizes at a radial distance $R$ within the gravitational field. Then according to the measurements of the laboratory instruments located outside the gravitational field, the Equation (27) is modified according to relation:

$$
\frac{1}{2} h n v_{n}=\frac{m e^{4}}{2 \hbar^{2}} \frac{1}{n^{2}}
$$

According to the clock of the above laboratory, following the relevant wording in [4] (section 4, Gravitational Time Dilation and Redshift, Equation (18)), the frequency $v_{n}$ is given by the equation:

$$
v_{n}=v_{n_{o}}\left(1-\frac{4 G M}{R c_{o}^{2}}\right)^{1 / 4}
$$

It is obvious that the first member of the Equation (32), is not equal to the average kinetic energy, according to the definition of kinetic energy given in the previous section, hence the Equation (32) no longer gives us the average kinetic energy. However, since the hydrogen atom is at a radial distance $R$ inside the gravitational field, using a clock located at the same radial distance, according to the estimated time given by the relation (23), the estimated frequency, $v_{n_{g}}$, is calculated according to the equation:

$$
v_{n_{g}}=v_{n}\left(1-\frac{4 G M}{R c_{o}^{2}}\right)^{-1 / 4}
$$

This estimated frequency is equal to the frequency $v_{n_{o}}$, measured when the effect occurs outside the gravitational field ${ }^{4}$. Therefore the mean value of kinetic energy is given by the equation:

$$
\overline{E_{k i n, n}}=\frac{1}{2} h n v_{n_{g}}=\frac{m e^{4}}{2 \hbar^{2}} \frac{1}{n^{2}}\left(1-\frac{4 G M}{R c_{o}^{2}}\right)^{-1 / 4}
$$

From the relations (15) and (24) the following equality results:

$$
m e^{4}=m_{o} e_{o}^{4}\left(1-\frac{4 G M}{R c_{o}^{2}}\right)^{1 / 4}
$$

therefore the average kinetic energy of the electron of the hydrogen atom at the energy level $n$ is:

$$
\overline{E_{k i n, n}}=\frac{m_{o} e_{o}^{4}}{2 \hbar^{2}} \frac{1}{n^{2}}
$$

\footnotetext{
${ }^{4}$ A similar description of this estimated frequency can be found in [4], section 4, Gravitational Time Dilation and Redshift, Equation (23).
} 
The total energy of the electron is the sum of the time average value of kinetic energy, time average value of Coulomb energy, and gravitational potential energy. The time average of Coulomb energy is denoted by $\overline{E_{d y n}}$. Since the gravitational dynamic energy is constant, given that the radial distance $R$ is constant, does not contribute to the energy difference of an energy transition of the electron, and, based on the virial theorem, $2 \overline{E_{k i n}}+\overline{E_{d y n}}=0$ ([11], paragraph 3-4, THE VIRIAL THEOREM), it follows that the energy of the electron, which actually contributes to the energy of an emitted or absorbed photon, is given by the relation:

$$
E_{n}=-\frac{m_{o} e_{o}^{4}}{2 \hbar^{2}} \frac{1}{n^{2}}
$$

that is, it is equal to the energy of the electron when the phenomenon takes place outside the gravitational field.

We now assume an energy transition of the electron of the hydrogen atom, from the initial energy level, $E_{n_{1}}$, with quantum number $n_{1}$ to the final energy level, $E_{n_{2}}$, with quantum number $n_{2}$, so, for the emission of a photon the inequality $n_{1}>n_{2}$ must apply.

The energy difference is calculated according to the known relation:

$$
\Delta E=E_{n_{2}}-E_{n_{1}}=-\frac{m_{o} e_{o}^{4}}{2 \hbar^{2}}\left(\frac{1}{n_{2}^{2}}-\frac{1}{n_{1}^{2}}\right)
$$

The estimated, by the clock of the electron, energy of the emitted photon, as stated in [4] (section 4, Gravitational Time Dilation and Redshift, Equation (25)), is given by the relation:

$$
\mathcal{E}_{p h_{g}}=h v_{o}\left(1-\frac{4 G M}{R c_{o}^{2}}\right)^{-1 / 4}
$$

where $h v_{o}=m_{p h_{o}} c_{o}^{2}$, and $v_{o}$ is the frequency of the emitted photon within the gravitational field, estimated by the clock of the electron. This frequency is equal to the frequency which is measured by a clock outside the gravitational field, when the emitted photon has come out of the gravitational field. Given that $\mathcal{E}_{p h_{g}}=-\Delta E$, the relations (39) and (40) give us:

$$
\frac{m_{o} e_{o}^{4}}{2 \hbar^{2}}\left(\frac{1}{n_{2}^{2}}-\frac{1}{n_{1}^{2}}\right)=h v_{o}\left(1-\frac{4 G M}{R c_{o}^{2}}\right)^{-1 / 4}
$$

If this phenomenon had taken place outside the gravitational field, then the energy of the emitted photon, estimated by a clock outside the gravitational field, would had been equal to:

$$
\frac{m_{o} e_{o}^{4}}{2 \hbar^{2}}\left(\frac{1}{n_{2}^{2}}-\frac{1}{n_{1}^{2}}\right)=h v_{o, n_{1} \rightarrow n_{2}}
$$

where $v_{o, n_{1} \rightarrow n_{2}}$ is the frequency of the emitted photon, estimated by a clock outside the gravitational field. From the relations (41) and (42) it appears that the photon whose emission takes place inside the gravitational field, when it has 
come out of the gravitational field, it has shifted toward the red in relation to the photon emitted outside the gravitational field, according to the following relation:

$$
v_{o}=v_{o, n_{1} \rightarrow n_{2}}\left(1-\frac{4 G M}{R c_{o}^{2}}\right)^{1 / 4} \simeq v_{o, n_{1} \rightarrow n_{2}}\left(1-\frac{G M}{R c_{o}^{2}}\right)
$$

\section{Experimental Confirmation of the Hypothesis of the Absolute Reference System}

In this section we examine the known experimental tests of the general theory of relativity, in order to determine whether the experimental results confirm the hypothesis of the absolute reference system.

Everything in this section is a brief description of what is stated in [4], section 4 , and section 5 .

\subsection{Gravitational Time Dilation}

According to the hypothesis of the absolute reference system, the bound photons, which are the building elements of the elementary particles of matter, will undergo these changes in frequency and wavelength. If the closed orbit of a bound photon has a length equal to $n \lambda$, where $n=1,2, \cdots$, then the time of a period is $T=n \lambda / c$, while, outside the gravitational field, the corresponding time is $T_{o}=n \lambda_{o} / c_{o}$. Therefore:

$$
T=T_{o} \frac{c_{o} \lambda}{c \lambda_{o}}=T_{o}\left(1-\frac{4 G M}{r c_{o}^{2}}\right)^{-1 / 4}
$$

According to the hypothesis of the absolute reference system, the estimated time is inversely proportional to the time of the aforementioned period ([1], 2.4.1. Contraction of Length and Time, p. 440-441). Assuming that $t_{g}$ is the estimated time recorded between two events occurring inside the gravitational field using a clock at a fixed position $\boldsymbol{r}$ also inside the gravitational field, and $t$ is the corresponding estimated time using a clock outside the gravitational field, then the correlation of these times is given by the equation:

$$
\frac{t_{g}}{t}=\frac{T_{o}}{T} \Leftrightarrow t_{g}=t\left(1-\frac{4 G M}{r c_{o}^{2}}\right)^{1 / 4} \simeq t\left(1-\frac{G M}{r c_{o}^{2}}\right)
$$

The corresponding result of the general theory of relativity is:

$$
t_{g}=t\left(1-\frac{2 G M}{r c_{o}^{2}}\right)^{1 / 2} \simeq t\left(1-\frac{G M}{r c_{o}^{2}}\right)
$$

Therefore the estimated time, based on the hypothesis of the absolute reference system, with a very good approximation is the same as that of the general theory of relativity. This estimated time value has been experimentally confirmed ([12] [13]). 


\subsection{The Advance of the Perihelion of Mercury}

Denoting the mass of the Sun by $M$ and the mass of Mercury by $m$, according to the Equation (15) and the relevant study in [4] (section 3, Mass and Velocity of a Photon in the Gravitational Field, Equation (15)), we get the equation:

$$
\frac{\mathrm{d}^{2} u}{\mathrm{~d} \theta^{2}}+u=\frac{G M m_{o}^{2}}{L^{2}}\left(1-\frac{4 G M}{r c_{o}^{2}}\right)^{-3 / 2}
$$

Given that $u=1 / r$, and $\left(1-4 G M /\left(r c_{o}^{2}\right)\right)^{-3 / 2} \simeq 1+6 G M /\left(r c_{o}^{2}\right)$, the first order approach is given by the differential equation:

$$
\frac{\mathrm{d}^{2} u}{\mathrm{~d} \theta^{2}}+\left(1-\frac{6 G^{2} M^{2} m_{o}^{2}}{c_{o}^{2} L^{2}}\right) u=\frac{G M m_{o}^{2}}{L^{2}}
$$

We define the constants:

$$
\begin{gathered}
A=1-\frac{6 G^{2} M^{2} m_{o}^{2}}{c_{o}^{2} L^{2}} \\
B=\frac{G M m_{o}^{2}}{L^{2}}
\end{gathered}
$$

The solution of the differential Equation (48) has the form:

$$
u=\frac{1}{r}=\frac{B}{A}+K \cos (\sqrt{A} \theta)
$$

where $K$ is constant.

Two consecutive minimizations of the distance $r$ are performed for $\sqrt{A} \theta=0$ and for $\sqrt{A} \theta=2 \pi$. For $\sqrt{A} \theta=0$ the angle $\theta$ is zero, while for $\sqrt{A} \theta=2 \pi$ the angle $\theta$ is given by the relation:

$$
\theta=\frac{2 \pi}{\sqrt{A}} \simeq 2 \pi+\frac{6 \pi G^{2} M^{2} m_{o}^{2}}{c_{o}^{2} L^{2}}
$$

This result is the same as that of the general theory of relativity ([14], $\$ 101$. Motion in a centrally symmetric gravitational field) and is confirmed by observations already announced in the 19th century.

More details on this topic are given in [4], subsection 5.1, The Advance of the Perihelion of Mercury.

\subsection{The Deflection of Light in the Gravitational Field of the Sun}

When a photon enters the gravitational field of the sun, its energy changes from the value $m_{p h_{o}} c_{o}^{2}$ to the value $m_{p h} c^{2}$, when it leaves the gravitational field, it takes again the value $m_{p h_{o}} c_{o}^{2}$. Also the motion of said photon at areat distance from the sun, where it can be considered to be outside the gravitational field, tends to be asymptotically a straight line, while inside the gravitational field it is curvilinear, due to the effect of the gravitational force of the Sun. Since the force is central, the trajectory equation has the geometric shape of the hyperbola, so, in polar coordinates $r, \Phi$, obeys the equation: 


$$
\frac{1}{r}=\frac{1}{a\left(\varepsilon^{2}-1\right)}+\frac{\varepsilon}{a\left(\varepsilon^{2}-1\right)} \cos \Phi
$$

where $\varepsilon$ is the eccentricity, which is greater than unit, and $a$ is the distance from the center $C$ to the vertex $V$ called the semi-major axis (Figure 2).

Holding only the first order terms, the approximate solution of the following differential equation:

$$
\frac{\mathrm{d}^{2} u}{\mathrm{~d} \theta^{2}}+u=\frac{G M m_{p h_{o}}^{2}}{L^{2}}\left(1-\frac{4 G M}{r c_{o}^{2}}\right)^{-3 / 2}
$$

as stated in [4] (subsection 5.2, The Deflection of Light in the Gravitational Field of the Sun, Equation (43)), is given by the equation:

$$
u=\frac{1}{r}=\frac{B^{\prime}}{A^{\prime}}+K^{\prime} \cos \left(\sqrt{A^{\prime}} \theta\right)
$$

where $A^{\prime}, B^{\prime}$ are the constants given by the equations:

$$
\begin{gathered}
A^{\prime}=1-\frac{6 G^{2} M^{2} m_{p h_{o}}^{2}}{c_{o}^{2} L^{2}} \\
B^{\prime}=\frac{G M m_{p h_{o}}^{2}}{L^{2}}
\end{gathered}
$$

The deflection angle is given by the equation:

$$
\delta \simeq \frac{2}{\varepsilon}
$$

We get the result:

$$
\delta \simeq 2^{\prime \prime} .13
$$

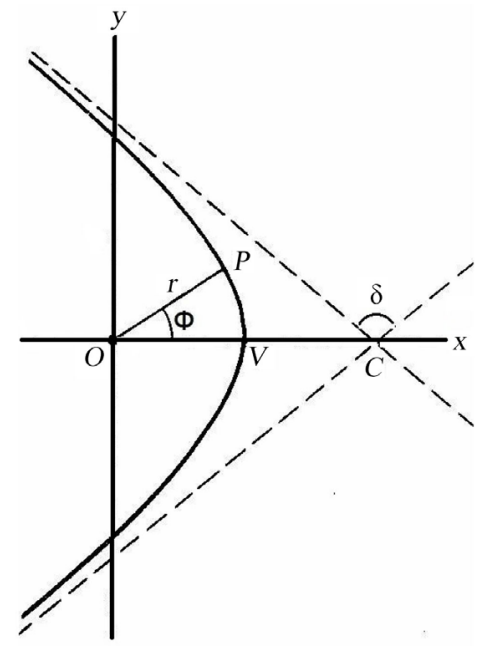

Figure 2. The continuous curved line is the elliptical orbit of the photon within a gravitational field. The hyperbolic orbit asymptotically approaches the dashed lines which are the directions of motion of the photon at a great distance from the spherical body of mass $M$. The angle $\delta$ is the angle of deflection of the photon. 
The corresponding value derived from the general theory of relativity is:

$$
\delta_{\mathrm{GR}}=\frac{4 G M}{R_{\varepsilon} c_{o}^{2}} \simeq 1^{\prime \prime} .75
$$

The result of calculating the deviation angle based on the hypothesis of the absolute reference system, which is $2 " .13$, is closer to the experimental value than the result of the general theory of relativity. This experimental value was obtained from measurements at the Sobral in 1919 ([15], V. General Conclusions. p. 330), which were:

From declinations 1 ".94

From right ascensions $2 " .06$

More details on this topic can be found in [4], subsection 5.2, The Deflection of Light in the Gravitational Field of the Sun.

\subsection{The Time Delay of Light}

We will now study the test proposed by Irwin I. Shapiro in order to measure a time delay (Shapiro delay) in the round-trip travel time for radar signals reflecting off other planets ([16]), sometimes called the fourth "classical test" of general relativity.

We consider that an electromagnetic signal is emitted from Earth, reflected on a planet (or spacecraft) and returned to Earth, but under the influence of the Sun's gravitational field during its motion towards the planet and during its return to the Earth. We will calculate the time delay due to the effect of the solar gravitational field on an elementary photonic signal, i.e. a photon. The path of the examined photon is the path $\mathrm{CBABC}$, of the axis $\mathrm{x}$ shown in Figure 3. Of course the deflection angle, which was calculated in the previous section, is very small, so the above-mentioned orbit is considered straight. Here we have ignored the motion of the Earth and planets during the round trip of the signal, because the corresponding velocities, estimated in the reference frame of the solar system, are much slower than the speed of light in vacuum.

The total time of the round trip path is calculated according to relation:

$$
\Delta t=\frac{2\left(x_{e}+x_{p}\right)}{c_{o}}+\frac{4 G M}{c_{o}^{3}} \ln \left(\frac{\left(r_{e}+x_{e}\right)\left(r_{p}+x_{p}\right)}{d^{2}}\right)
$$

This result agrees with the corresponding result of the general theory of relativity ([17], 7.2 The Time Delay of Light, Equation (7.31)).

More details on this topic can be found in [4], subsection 5.3, The Time Delay of Light.

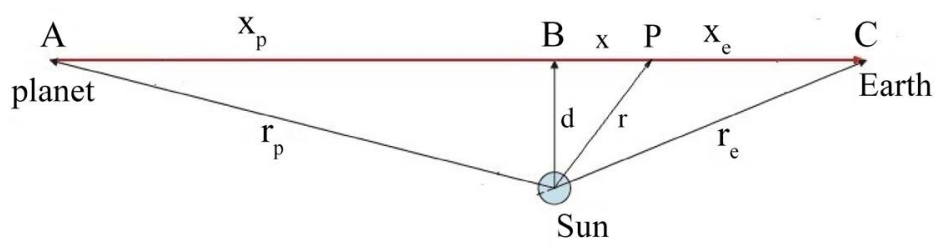

Figure 3. Geometry of light deflection measurements. 


\subsection{The Gravitational Redshift}

We will first study the redshift due to the Earth's gravitational field for example, which is observed if two measurements of the estimated energy of a photon are taken at two different radial distances $r_{1}$ and $r_{2}$ that differ only slightly, and $r_{1}<r_{2}$. Also we consider that $r_{1}$ is equal to the distance of the earth ground of the experiment from the center of the Earth. The ratio of the two estimated energies is calculated as follows:

$$
\frac{\mathcal{E}_{p h_{g}}\left(r_{2}\right)}{\mathcal{E}_{p h_{g}}\left(r_{1}\right)}=\frac{m_{p h_{o}} c_{o}^{2}\left(1-\frac{4 G M}{r_{2} c_{o}^{2}}\right)^{-1 / 4}}{m_{p h_{o}} c_{o}^{2}\left(1-\frac{4 G M}{r_{1} c_{o}^{2}}\right)^{-1 / 4}} \simeq 1-\frac{G M}{c_{o}^{2}} \frac{r_{1}-r_{2}}{r_{1} r_{2}}
$$

If $h=r_{2}-r_{1}$ is the height, $R$ is the radius of the Earth, $g=G M / R^{2}$, and $r_{1} r_{2} \simeq R^{2}$, then the previous relation becomes:

$$
\frac{\mathcal{E}_{p h_{g}}\left(r_{2}\right)}{\mathcal{E}_{p h_{g}}\left(r_{1}\right)} \simeq 1-\frac{g h}{c_{o}^{2}}
$$

This result agrees with the corresponding prediction of the general theory of relativity and has been experimentally confirmed ([18]).

More details on this topic can be found in [4], subsection 5.4, The Gravitational Redshift.

\section{Conclusion}

The physics of an absolute reference system is a comprehensive and self-contained view of physical reality, extending to all areas of the physical sciences, and confirmed by a wide range of scientific observations and experiments, including all experiments performed from time to time in order to confirm the special and general theory of relativity. In this article, the quantum gravitational phenomena are studied, and it is proved that the gravitational redshift is fully interpreted based on the principles of the absolute reference system hypothesis. Also, the calculations are confirmed by the experimental results, and it is also found that the prediction for the experiment on the deflection of light in the gravitational field of the Sun on the basis of the hypothesis of the absolute reference system, is in better agreement with the experimental data compared to the corresponding prediction of the general theory of relativity. It is therefore necessary to carry out an experiment in order to take high-precision measurements of light deflection, in the special case of the effect of the gravitational field of the Sun.

\section{Conflicts of Interest}

The author declares no conflicts of interest regarding the publication of this paper. 


\section{References}

[1] Patrinos, K. (2019) Journal of Applied Mathematics and Physics, 7, 431-475. https://doi.org/10.4236/jamp.2019.73033

[2] Patrinos, K. (2019) Journal of Applied Mathematics and Physics, 7, 747-780. https://doi.org/10.4236/jamp.2019.74052

[3] Patrinos, K. (2020) Journal of Applied Mathematics and Physics, 8, 999-1015. https://doi.org/10.4236/jamp.2020.86078

[4] Patrinos, K. (2021) Journal of Applied Mathematics and Physics, 9, 1194-1214. https://doi.org/10.4236/jamp.2021.96082

[5] Einstein, A. (1907) Jahrbuch der Radioaktivität und Elektronik, 4, 411.

[6] Einstein, A. (1915) Die Feldgleichungen der Gravitation. Sitzungsberichte der Preußischen Akademie der Wissenschaften zu Berlin. 844-847.

[7] Einstein, A. (1916) Annalen der Physik, 354, 769-822. https://doi.org/10.1002/andp.19163540702

[8] Einstein, A. (1916) Näherungsweise Integration der Feldgleichungen der Gravitation. Sitzungsberichte der Königlich Preußischen Akademie der Wissenschaften Berlin (Part 1). 688-696.

[9] Einstein, A. (1917) Kosmologische Betrachtungen zur allgemeinen Relativitätstheorie. Sitzungsberichte der Preußischen Akademie der Wissenschaften. 142.

[10] Einstein, A. (1918) Über Gravitationswellen. Sitzungsberichte der Königlich Preußischen Akademie der Wissenschaften Berlin (Part 1). 154-167.

[11] Goldstein, H. (1980) Classical Mechanics. Second Edition, Addison-Wesley Publishing Company, Boston.

[12] Hafele, J.C. and Keating, R.E. (1972) Science, 177, 166-168. https://doi.org/10.1126/science.177.4044.166

[13] Hafele, J.C. and Keating, R.E. (1972) Science, 177, 168-170. https://doi.org/10.1126/science.177.4044.168

[14] Landau, L. and Lifshitz, E. (1975) The Classical Theory of Fields. Pergamon Press Ltd., Oxford.

[15] Dyson, F.W., Eddington, A.S. and Davidson, C. (1920) Philosophical Transactions of the Royal Society of London. Series A, Containing Papers of a Mathematical or Physical Character, 220, 291-333. https://doi.org/10.1098/rsta.1920.0009

[16] Shapiro, I.I. (1964) Physical Review Letters, 13, 789-791. https://doi.org/10.1103/PhysRevLett.13.789

[17] Will, C.M. (1993) Theory and Experiment in Gravitational Physics. Cambridge University Press, Cambridge.

[18] Pound, R.V. and Snider, J.L. (1965) Physical Review, 140, B788. https://doi.org/10.1103/PhysRev.140.B788 